\title{
Atenção farmacêutica na Doença de Alzheimer
}

\author{
Pharmaceutical care in Alzheimer's Disease \\ Atención farmacéutica en la Enfermedad de Alzheimer
}

Patrícia Rayanne

ORCID: https://orcid.org/0000-0002-2006-7208

Centro Universitário Santo Agostinho, Brasil E-mail: patriciarayanne660@gmail.com

Lenara Veras

ORCID: https://orcid.org/0000-0002-2016-5593 Centro Universitário Santo Agostinho E-mail: lenaraveras16@gmail.com

Joseana Martins Soares de Rodrigues Leitão

ORCID: https://orcid.org/0000-0001-8043-3663

Centro Universitário Santo Agostinho, Brasil E-mail: joseanaleitao@hotmail.com

\begin{abstract}
Resumo
A Doença de Alzheimer (DA) é um distúrbio neurodegenerativo e principal causa de demência, pois a doença afeta inicialmente o hipocampo e progride lentamente para outras partes do cérebro, causando dano celular e morte, especificamente de neurônios, gerando danos irreversíveis e consequências para todo o funcionamento cognitivo, afetiva, intelectual e comportamental, influenciando negativamente na autonomia e independência do indivíduo. Os pacientes são submetidos a tratamentos de uso crônico, visto que não há cura para a doença de Alzheimer. O tratamento visa minimizar os sintomas da doença, possibilitando melhora parcial para prolongar a sobrevida e retardar a progressão. Dada a real importância da assistência farmacêutica dirigida ao paciente, que otimiza a resposta de uma determinada farmacoterapia, por meio da qual o farmacêutico juntamente com o paciente, realiza um plano de acompanhamento. Nesse contexto, este trabalho tem como objetivo salientar a importância da atenção farmacêutica tanto na farmacoterapia quanto fora da mesma. Trata-se de um estudo de revisão integrativa transversal e descritiva com abordagem qualitativa e quantitativa sobre o tema, onde foram feitas buscas nas bases de dados Scielo e BVS acerca do tema. Foram encontrados 1.199 trabalhos, no qual foi feita uma triagem para a coleta dos resultados. Foi observado que o tratamento da Doença de Alzheimer é capaz de retardar ou estagnar, temporariamente, o avanço dos sintomas visando aliviar os déficits cognitivos, as alterações de comportamento e melhorar a qualidade de vida do portador e de sua família. O tratamento da Doença de Alzheimer deve ser multidisciplinar e com o objetivo de propiciar a estabilização do comprometimento cognitivo, modificando as manifestações da doença, com um mínimo de efeitos adversos. Nesse contexto, os imunomoduladores têm maior probabilidade de se tornarem drogas capazes de influenciar o curso da DA, pois os estudos selecionados apresentaram melhor qualidade e os resultados foram promissores. Por outro lado, a toxicidade das drogas para o tratamento da DA constitui um grande obstáculo, e para contornar tal situação a presença da atenção farmacêutica se torna cada vez mais imprescindível.
\end{abstract}

Palavras-chave: Doença de Alzheimer; Atenção Farmacêutica; Idoso.

\begin{abstract}
Alzheimer's Disease (AD) is a neurodegenerative disorder and the main cause of dementia, as the disease initially affects the hippocampus and slowly progresses to other parts of the brain, causing cell damage and death, specifically of neurons, generating irreversible damage and consequences for all cognitive functioning. , affective, intellectual and behavioral, negatively influencing the individual's autonomy and independence. Patients are undergoing treatments for chronic use, as there is no cure for Alzheimer's disease. Treatment aims to minimize the symptoms of the disease, enabling partial improvement to prolong survival and delay progression. Given the real importance of pharmaceutical assistance directed to the patient, which optimizes the response of a given pharmacotherapy, through which the pharmacist, together with the patient, carries out a follow-up plan. In this context, this work aims to highlight the importance of pharmaceutical care both in pharmacotherapy and outside it. This is a cross-sectional and descriptive integrative review study with a qualitative and quantitative approach on the subject, where searches were made in the Scielo and VHL databases about the subject. A total of 1,199 works were found, in which a screening was carried out to collect the results. It was observed that the treatment of Alzheimer's Disease is capable of delaying or temporarily stagnating the advance of symptoms, aiming to alleviate cognitive deficits, behavioral changes and improve the quality of life of the patient and his family. The treatment of Alzheimer's Disease must be multidisciplinary and with the objective of providing stabilization of cognitive impairment, modifying the manifestations of the disease, with a
\end{abstract}


minimum of adverse effects. In this context, immunomodulators are more likely to become drugs capable of influencing the course of $\mathrm{AD}$, as the selected studies had better quality and the results were promising. On the other hand, the toxicity of drugs for the treatment of $\mathrm{AD}$ is a major obstacle, and to overcome this situation, the presence of pharmaceutical care becomes increasingly essential.

Keywords: Alzheimer's Disease; Pharmaceutical attention; Old man.

\section{Resumen}

La Enfermedad de Alzheimer (EA) es un trastorno neurodegenerativo y la principal causa de demencia, ya que la enfermedad afecta inicialmente al hipocampo y progresa lentamente a otras partes del cerebro, provocando daño celular y muerte, específicamente de neuronas, generando daños y consecuencias irreversibles para todo el funcionamiento cognitivo. , afectiva, intelectual y conductual, influyendo negativamente en la autonomía e independencia del individuo. Los pacientes se someten a tratamientos de uso crónico, ya que no existe cura para la enfermedad de Alzheimer. El tratamiento tiene como objetivo minimizar los síntomas de la enfermedad, permitiendo una mejoría parcial para prolongar la supervivencia y retrasar la progresión. Dada la importancia real de la asistencia farmacéutica dirigida al paciente, que optimiza la respuesta de una determinada farmacoterapia, mediante la cual el farmacéutico, junto con el paciente, realiza un plan de seguimiento. En este contexto, este trabajo tiene como objetivo resaltar la importancia de la atención farmacéutica tanto en la farmacoterapia como fuera de ella. Se trata de un estudio de revisión transversal, descriptivo, integrador, con abordaje cualitativo y cuantitativo sobre el tema, donde se realizaron búsquedas en las bases de datos Scielo y BVS sobre el tema. Se encontraron un total de 1.199 trabajos, en los que se realizó un cribado para recoger los resultados. Se observó que el tratamiento de la Enfermedad de Alzheimer es capaz de retrasar o estancar temporalmente el avance de los síntomas, con el objetivo de paliar déficits cognitivos, cambios de comportamiento y mejorar la calidad de vida del paciente y su familia. El tratamiento de la Enfermedad de Alzheimer debe ser multidisciplinar y con el objetivo de proporcionar la estabilización del deterioro cognitivo, modificando las manifestaciones de la enfermedad, con un mínimo de efectos adversos. En este contexto, es más probable que los inmunomoduladores se conviertan en fármacos capaces de influir en el curso de la EA, ya que los estudios seleccionados tuvieron mejor calidad y los resultados fueron prometedores. Por otro lado, la toxicidad de los fármacos para el tratamiento de la EA es un gran obstáculo, y para superar esta situación, la presencia de cuidados farmacéuticos se vuelve cada vez más imprescindible.

Palabras clave: Enfermedad de Alzheimer; Atención farmacéutica; Anciano.

\section{Introdução}

A Doença de Alzheimer (DA) é um transtorno neurodegenerativo e principal causa de demência, pois inicialmente a doença atinge o hipocampo, estrutura cerebral responsável pela memória, e lentamente progride para outras partes cerebrais, causando lesão e morte celular, especificamente dos neurônios, gerando danos irreversíveis e consequências em todo o funcionamento cognitivo, afetivo, intelectual e comportamental, influenciando negativamente na autonomia e independência do indivíduo (Filho \& Barreira, 2017).

Tendo em conta o impacto deletério da Doença de Alzheimer nas capacidades dos portadores, que perdem a autonomia nas atividades da vida diária e se tornam dependentes de terceiros, as repercussões sentidas no doente, família e comunidade, assim como as consequências econômicas, torna-se fundamental a existência de um tratamento modificador da doença ou, idealmente, preventivo (Ferreria \& Massano, 2013).

Os portadores são submetidos a tratamentos de uso crônico, haja vista que não existe cura para a DA. O tratamento visa minimizar os sintomas da doença, possibilitando uma melhora parcial, a fim de prolongar a sobrevida e retardar o tempo de progressão (Brasil, 2017). Os avanços na área da saúde têm possibilitado uma maior sobrevida e qualidade de vida dos portadores, que devem receber um tratamento de caráter multidisciplinar, visto que se trata de uma doença que envolve sinais e sintomas de diferentes magnitudes com peculiaridades de condutas (Filho \& Barreira, 2017).

Tendo em vista a real importância do cuidado farmacêutico voltada para o paciente idoso com doença de Alzheimer, a Atenção Farmacêutica otimiza a resposta de determinada farmacoterapia, pelos quais o farmacêutico juntamente ao portador, realiza um plano de seguimento. O processo de construção de um plano de atenção farmacoterapêutica é basicamente composto por um conjunto de ações de coleta de dados, análise e estudo do estado de situação, geração informações, tomadas de decisões e realização de avaliações e intervenções clínicas a partir de um plano realizado junto ao portador, sempre 
documentando os resultados (Madureira et al., 2018).

Com este plano, objetiva-se determinar a necessidade do indivíduo com Alzheimer e usuário de uma farmacoterapia que se encontra em situações singulares devido aos sinais e sintomas casuais da doença, além das comorbidades, para fornecer serviços antes, durante e após o tratamento farmacológico de modo a assegurar a adesão, a segurança e a eficácia.

\section{Metodologia}

Este estudo é uma revisão integrativa da literatura de caráter e descritivo com abordagem qualitativa e quantitativa que reúne informações relevantes sobre uma temática definida e discute os resultados observados com fundamento científico. Uma revisão integrativa sintetiza resultados obtidos em pesquisas, de maneira ordenada e abrangente, integrando o conhecimento sobre um determinado tema.

Foram selecionados artigos científicos, publicados entre 2012 e 2018. As buscas por estudos originais foram feitas utilizando as bases de dados: Medical Literature Analysis and Retrieval System Online (MEDLINE/PubMed), Scientific Electronic Library Online (SciELO), Biblioteca Virtual em Saúde (BVS) e Literatura Latino-Americana e do Caribe em Ciências da Saúde (LILACS). Foram utilizados os seguintes Descritores em Ciências da Saúde, que constam na plataforma DeCS da Biblioteca Virtual de Saúde: doença de Alzheimer, atenção farmacêutica e idoso. A coleta de dados foi realizada entre abril de 2021 a setembro de 2021.

Tabela 1 - Número de artigos encontrados nas bases de dados com os descritores da pesquisa.

\begin{tabular}{lll}
\hline \multicolumn{1}{c}{ Descritores } & Bases de Dados & $\mathbf{N}^{\circ}$ de artigos \\
\hline Alzheimer e Atenção Farmacêutica e & BVS & 1.134 \\
Idosos & & \\
\hline Alzheimer e Atenção Farmacêutica e & SCIELO & 65 \\
Idosos & & 1.199 \\
\hline \multicolumn{1}{c}{ TOTAL } & & \\
\hline
\end{tabular}

Fonte: Dados da pesquisa (2021).

Observa-se que, através dos descritores Alzheimer e Atenção Farmacêutica e Idosos 1.199 artigos na base de dados BVS e 65 artigos na base de dados SCIELO, totalizando 1.199 artigos. Para seleção dos artigos científicos condizentes com o propósito do estudo, dentre os encontrados, foram utilizados os seguintes critérios de inclusão: publicação entre 2012 a 2018 , trabalhos disponíveis na íntegra, gratuitos, em português, inglês e espanhol, artigos de intervenção, quase experimental, análises, estudo de caso e estudos transversais. Foram excluídos do estudo resumos, teses, dissertações e monografias..

\section{Resultados e Discussão}

\section{Doença de Alhzeimer}

A doença de Alzheimer é uma condição neurodegenerativa e progressiva, caracterizada por deterioração da memória e de outras funções cognitivas com comprometimento progressivo das atividades de vida diária e uma multiplicidade de alterações comportamentais e psicológicos que comprometem a qualidade de vida na velhice (Araújo, Tascarollo, Antonio, 2019).

Os sintomas da Doença de Alzheimer incluem perda progressiva de memória, comprometimento cognitivo, como dificuldade em resolver problemas e desorientação no tempo e no espaço, entre outros, em uma população envelhecida que 
causa danos graves aos neurônios colinérgicos em uma área específica do cérebro, o hipocampo (Cazarim et al., 2016). As mudanças cerebrais acontecem antes do surgimento de sintomas de demência, as lesões se manifestam principalmente no córtex cerebral e influenciam na diminuição do tamanho do cérebro (Allegri, 2020).

A etiologia da doença permanece indefinida, embora um progresso considerável tenha sido alcançado para a compreensão de mecanismos bioquímicos e genéticos. O principal mecanismo fisiopatológico subjacente à Doença de Alzheimer é o acúmulo anormal de peptídeo $\beta$-amilóide (A $\beta$ ) em placas senis (SP) e proteína tau hiperfosforilada (TP) em emaranhados neurofibrilares (NFT), resultando em atrofia cerebral (Moreira, Jansen, Silva, 2020).

Todavia, existem hipóteses que doença está associada a uma redução dos níveis corticais cerebrais de diversas proteínas e neurotransmissores, em especial a acetilcolina e sua enzima de síntese a colina-acetiltransferase (CAT). Ocorre também aumento da perda dos neurônios glutaminérgicos, com distúrbios nos receptores N-metil-D-aspartato, NMDAreceptor glutaminérgico. A colina acetiltransferase é a enzima principal para síntese de acetilcolina, e uma redução nas concentrações de colina acetiltransferase sugere uma diminuição no número de neurônios colinérgicos presentes (Filho \& Barreira, 2017).

O comprometimento cognitivo é detectado e diagnosticado mediante a combinação de anamnese com paciente e informante que tenha conhecimento da história do portador e de avaliação cognitiva objetiva, mediante exame breve do estado mental ou avaliação neuropsicológica. A avaliação neuropsicológica deve ser realizada quando a anamnese e o exame cognitivo breve realizado pelo médico não forem suficientes para permitir um diagnóstico confiável. Um exame de imagem cerebral, tomografia computadorizada (TC) ou ressonância magnética (RM), é útil para excluir lesões estruturais que podem contribuir para a demência (Brasil, 2017).

O processo de investigação diagnóstica para preencher os critérios inclui história completa (com portador e cuidador), avaliação clínica incluindo a escala de avaliação clínica da demência, rastreio cognitivo - testes cognitivos como o MiniExame do Estado Mental (MEEM), exames laboratoriais - hemograma, eletrólitos (sódio, potássio, cálcio), glicemia, ureia e creatinina, TSH e alanino-aminotransferase (ALT/TGP), aspartato-aminotransferase (AST/TGO), vitamina B12, ácido fólico -, sorologia sérica para sífilis (VDRL) e HIV (em portadores com menos de 60 anos), e imagem cerebral (TC sem contraste ou RM) (Brasil, 2017).

A sobrevivência após o diagnóstico varia consideravelmente e depende de vários fatores em complexa interação, de forma que a expectativa de vida diminui quanto maior a idade do diagnóstico e quanto maior a gravidade e perda da funcionalidade.

\section{Desafio de cuidadores de portadores acometidos pela Doença de Alzheimer}

A Doença de Alzheimer é uma das mais importantes a acometer os idosos. Esta demência relaciona-se a um declínio funcional e perda de autonomia causando aos seus portadores dependência de outros indivíduos (Martins et al., 2019).

Além de prejudicar o funcionamento biológico do indivíduo, a Doença de Alzheimer pode ser considerada uma doença social, uma vez que a falta de conhecimento sobre as condições gerais da doença acarreta preconceitos que atingem a família do doente, causando um ônus crescente sobre o idoso e a família (Pereira \& Oliveira, 2019).

Desse modo os estudos advertem que para que o cuidador tenha condições de se reorganizarem nos âmbitos pessoal, familiar e social é essencial à aquisição de informações e os apoios adequados. A partir destes subsídios poderá conciliar as suas obrigações de trabalho com o cuidado, pois cabe a eles dar a continuidade dos cuidados básicos que o indivíduo acometido pela doença necessita no seu processo de vida (Martins et al., 2019). O ato de cuidar pode acarretar o desgaste biopsicossocial do cuidador e por outro lado à proximidade dos cuidados com o indivíduo com a Doença de Alzheimer, pode provocar a redescoberta da união familiar, bem como a valorização do ato de cuidar (Fernandes \& Andrade, 2017). 
Em decorrência da demanda do cuidado ofertado a idosos dependentes, prejuízos à saúde são relatados por esses profissionais, devido à existência de eventos estressores relativos ao ato de cuidar, que causam influência negativa na qualidade de vida dessas pessoas (Martins, et al., 2019). Desse modo, Antunes (2017) constatou que cerca de 87,9\% dos cuidadores apresentavam complicações na sua saúde, já que após assumirem a ocupação, evidenciaram pelo menos uma doença, podendo ser submetidos a transtornos psicológicos, tais como ansiedade, depressão e estresse.

Fatores como sentimento de sobrecarga decorrente da carga horária utilizada no cuidado do idoso, complexidade do cuidado e, por comumente desconsiderarem suas próprias necessidades, geram um aumento na probabilidade de esses indivíduos manifestarem sintomas depressivos e ansiosos (Martins, et al.; 2019).

Portanto, é fundamental o apoio direcionado não só para o idoso que recebe o cuidado, mas também ao cuidador, mediante ações que possibilitem a contemplação de suas necessidades individuais. Tal prática deve representar um mecanismo de suporte a essa população, para garantir não só a diminuição de sintomas depressivos, de ansiedade e estresse percebidos nos cuidadores, mas também, mas melhorar a qualidade do cuidado prestado ao idoso com Doença de Alzheimer (Martins, et al., 2019).

\section{Tratamento}

O tratamento da Doença de Alzheimer é capaz de retardar ou estagnar, temporariamente, o avanço dos sintomas, visando aliviar os déficits cognitivos, as alterações de comportamento e melhorar a qualidade de vida do portador e de sua família (Madureira, et al., 2018). O tratamento da Doença de Alzheimer deve ser multidisciplinar e com o objetivo de propiciar a estabilização do comprometimento cognitivo, modificando as manifestações da doença, com um mínimo de efeitos adversos (Brasil, 2017).

Tabela 2 - Características de substâncias modificadoras do curso da doença de Alzheimer

\begin{tabular}{|c|c|c|}
\hline Classe & Agente & Características \\
\hline \multirow{5}{*}{$\begin{array}{l}\text { Grupos com alvos terapêuticos não } \\
\text { esclarecidos em relação ao curso da doença }\end{array}$} & Inibidores da colinesterase & $\begin{array}{l}\text { Relação positiva da acetilcolinesterase com a } \\
\text { formação do peptídeo A } \beta \text { e densidade } \\
\text { aumentada, com subsequente deposição nos } \\
\text { neurônios }\end{array}$ \\
\hline & Terpenóides & $\begin{array}{l}\text { Capaz de reduzir os níveis de } \mathrm{A} \beta \text { e promover } \\
\text { sua degradação. Investigou a capacidade de } \\
\text { reduzir a produção de } \mathrm{A} \beta \text { no processo } \\
\text { modulado pelo APP }\end{array}$ \\
\hline & Imunomoduladores & $\begin{array}{l}\text { Demonstrou capacidade de coibir a deposição e } \\
\text { formação de toda placa e também auxiliar na } \\
\text { depuração do oligopeptídeo no SNC }\end{array}$ \\
\hline & Agentes para tratamento da diabetes & $\begin{array}{l}\text { Possível associação para reduzir a agregação de } \\
\mathrm{A} \beta \text { oligômeros por meio clivagem deste } \\
\text { peptídeo }\end{array}$ \\
\hline & Agentes modificadores de lipídios & $\begin{array}{l}\text { Promoveu a formação reduzida de placa } \mathrm{A} \beta \text { em } \\
\text { indivíduos com o alelo APOE e4. Considerado } \\
\text { como medicamentos multi-direcionados porque } \\
\text { baixam os níveis de colesterol no cérebro, têm } \\
\text { efeitos anti-inflamatórios propriedades e } \\
\text { melhorou a microcirculação, o que reduziu a } \\
\text { formação de NFTs }\end{array}$ \\
\hline
\end{tabular}


Research, Society and Development, v. 10, n. 13, e385101321247, 2021

(CC BY 4.0) | ISSN 2525-3409 | DOI: http://dx.doi.org/10.33448/rsd-v10i13.21247

\begin{tabular}{ll} 
Polifenóis & $\begin{array}{l}\text { Aumentou a depuração da proteína tau quando } \\
\text { não estruturada }\end{array}$ \\
\hline Agonistas muscarínicos & Capaz de modificar a concentração e agregação \\
& de A $\beta$ no líquido cefalorraquidiano, portanto \\
& diminuição da formação de NP \\
\hline Agonistas nicotínicos & Atuou no receptor nicotínico da acetilcolina; \\
& evitando assim a hiperfosforilação da proteína \\
& tau. \\
\hline Retinóides & Acúmulo suprimido de A $\beta$ e diminuição do \\
& processo de inflamação do cérebro \\
\hline Antagonistas de NMDA & Inibiu a neurotoxicidade do processo de \\
excitação causada pelo glutamato e reduziu \\
hiperfosforilação da proteína tau \\
Capaz de inibir o influxo de íons de cálcio nos \\
canais A A, evitando assim a apoptose e \\
sinalização desproporcional entre neurônios
\end{tabular}

Inibidores ou moduladores da gamma ou beta- Atividade enzimática inibida ou modulada, secretase controlando assim a produção de no cérebro e formação de agregados insolúveis

Inibidores da fosfodiesterase Diminuição da expressão gênica via cAMP que estava envolvida na produção da proteína tau e, portanto, processo de interrupção minimizado

Grupos com alvos terapêuticos alternativos Moduladores de ciclina dependentes de kinase Hiperfosforilação modulada da proteína tau por em relação ao curso da doença meio de um processo de quinase dependente de ciclina

Inibidores da fosfolipase A2 Diminuição da excitotoxicidade por meio da inibição da fosfolipase A2 e aumento da depuração de tau quando não era estruturado medicamentos por meio da mediação de processos inflamatórios causados por sinais pró-inflamatórios invocando citocinas que são características em regiões com placa $\mathrm{A} \beta$

\begin{tabular}{ll}
\hline Drogas nootrópicas & Aumento do desempenho cognitivo na DA \\
\hline Hormônioterapia & Proporcionou ação preventiva contra a doença \\
& de Alzheimer em mulheres submetidas a \\
& hormônios (terapia de reposição) \\
\hline Antioxidantes & Espécies reativas reduzidas de oxigênio (ROS), \\
& proporcionando assim a neuroproteção \\
\hline Agentes quelantes & Neurotoxicidade modulada causada por \\
& radicais livres
\end{tabular}

Possíveis modificadores do curso da doença com um alvo terapêutico específico

Fonte: Dados da pesquisa (2021).

As diretrizes estabelecidas nas terapias farmacológicas para o tratamento da Doença de Alzheimer podem ser resumidas como inibição da degradação da acetilcolina ou bloqueio dos receptores de glutamato. Isso tem o objetivo de 
aumentar a atividade colinérgica e diminuir a hiperatividade do neurotransmissor excitatório glutamato nas regiões do córtex e do hipocampo (Cazarim, et al., 2016).

Assim, os medicamentos comumente usados na prática clínica atual são os inibidores da acetilcolinesterase (IAChEs): donepezila, galantamina e rivastigmina, que podem ser usados isoladamente ou em combinação com a memantina, um antagonista dos receptores NMDA que também pode ser usado isoladamente, dependendo do estágio da doença (Filho \& Barreira, 2017).

Donepezila é um medicamento inibidor reversível e seletivo de colinesterase, e que se mostrou eficaz no tratamento da Doença de Alzheimer em doses de 5 e $10 \mathrm{mg}$. O tratamento inicial é de $5 \mathrm{mg} /$ dia e a dose pose ser aumentada a partir da quarta semana para $10 \mathrm{mg} /$ dia. Esse medicamento apresenta vantagem quando comparados a outros da mesma classe, pois é administrado uma vez ao dia (Almeida, 1998).

Galantamina é um inibidor reversível da ACHE e deve ser administrado uma vez ao dia, de preferência com alimentos, por se tratar de cápsulas de liberação prolongada. A dose inicial deve ser com $8 \mathrm{mg} / \mathrm{dia}$, por via oral, durante 4 semanas e a dose máxima é de $24 \mathrm{mg} /$ dia. Em caso de insuficiência hepática ou renal moderada, a dose deve ser ajustada considerando a dose máxima de $16 \mathrm{mg} / \mathrm{dia}$ (Brasil, 2017).

Rivastigmina é um inibidor de ACHE e da BACHE, o tratamento deve ser iniciado com doses de $3 \mathrm{mg} / \mathrm{dia}$ e a dose pode ser aumentada a partir da segunda semana, apresentando a dose máxima de $12 \mathrm{mg} / \mathrm{dia}$, utilizado duas vezes ao dia, pode apresentar-se também na forma de adesivos transdérmicos, com apresentação de $5 \mathrm{~cm}^{2}$ durante por pelo menos 4 semanas de tratamento, um método alternativo, podendo oferecer uma maior adesão ao tratamento (Filho \& Barreira, 2017).

Memantina é um antagonista de NMDA, o tratamento inicial deve ser de $5 \mathrm{mg} / \mathrm{dia}$ por via oral (1/2 comprimido), aumentar $5 \mathrm{mg} / \mathrm{semana}$ nas 3 semanas subsequentes até chegar à dose de $20 \mathrm{mg} / \mathrm{dia}$ (1 comprimido de 10mg duas vezes por dia) na quarta semana e manter esta dose (Brasil, 2017).

Os medicamentos para o tratamento da Doença de Alzheimer são disponibilizados gratuitamente aos usuários por meio do Componente Especializado da Atenção Farmacêutica (CEAF), e seu fornecimento está sujeito ao cumprimento dos critérios e normas estabelecidos pelo Protocolo Clínico e Diretrizes Terapêuticas (PCDT) da doença de Alzheimer (Almeidabrasil, et al., 2016).

Não podemos dispensar o tratamento não farmacológico e a sua extrema importância nos resultados positivos aos pacientes. A terapia não farmacológica coopera na melhora da cognição dos portadores, com isto se tem uma melhora significante na qualidade de vida dos idosos que lutam contra a Doença de Alzheimer (Carvalho, Magalhães, Pedroso, 2016).

A incentivação constante do idoso com Doença de Alzheimer com atividades físicas e mentais, participação em atividades sociais com outras pessoas, exercícios de memória e mesmo afazeres domésticos são estratégias relevantes na melhora de seu tratamento (Carvalho, Magalhães, Pedroso, 2016).

\section{Adesão ao tratamento de portadores da Doença de Alzheimer}

A adesão ao tratamento é o cumprimento de todas as orientações do profissional de saúde prescritor, a fim da terapêutica escolhida ser eficaz e eficiente. O cuidado para a realização de um tratamento geriátrico correto deve ser ampliado pela atenção do cuidador ou responsável, uma vez que qualquer dificuldade que o idoso possa ter com o tratamento poderá bloquear ou interromper a continuidade do mesmo (Filho \& Barreira, 2017).

A adesão ao tratamento farmacológico é uma preocupação e um obstáculo significativo à farmacoterapia ideal. Uma vez que é difícil prever o comportamento do portador quanto à adesão ao tratamento, é fundamental identificar os obstáculos à adesão que possam ser controlados ou modificados. A razão mais comum para a falta de adesão ao tratamento farmacológico é o esquecimento por parte do portador. Outras razões incluem: outras prioridades, decisão de omitir doses, falta de informações 
e fatores emocionais (Carvalho, Magalhães, Pedroso, 2016).

Existe uma dificuldade em decorrência da maioria dos portadores, fazer uso de múltiplos medicamentos, podendo complicar a administração entre eles. Além das limitações que, com tempo, a Doença de Alzheimer vai ocasionando às atividades de vida diária dos portadores.

Outras barreiras relacionadas para a não adesão envolvem dimensões, como: disponibilidade (isto é, volume de medicamentos ofertados e disponíveis de acordo com as necessidades do usuário); acessibilidade geográfica entendida como relação entre a localização do serviço e a localização dos usuários; acomodação (como os serviços são fornecidos e organizados para atender aos usuários); capacidade aquisitiva (capacidade do usuário em arcar com os custos do tratamento); e aceitabilidade (atitudes de profissionais de saúde e usuários em relação às características e práticas do serviço) (Almeida-brasil et al., 2016).

Inúmeros são os fatores que embaraçam a adesão ao tratamento, dessa forma devem ser avaliados pela atenção farmacêutica ao paciente e assim fazer o uso racional dos medicamentos.

\section{Atenção farmacêutica na Doença de Alzheimer}

A Atenção Farmacêutica é uma prática profissional que se baseia na provisão responsável da farmacoterapia, com a finalidade de alcançar resultados efetivos em resposta ao tratamento prescrito, e assim melhore a qualidade de vida do usuário. Busca resolver ou prevenir os problemas farmacoterapêuticos de forma documentada ou sistematizada. Além disso, engloba o acompanhamento do indivíduo visando responsabilizar-se junto deste, para que o fármaco prescrito seja eficaz e seguro, na posologia adequada e resulte no efeito terapêutico almejado (Moreira, Jansen, Silva, 2020).

A atenção farmacêutica trata-se em conjunto de ações desenvolvidas pelo farmacêutico e por outros profissionais de saúde voltadas à promoção, à proteção e à recuperação da saúde, tanto no nível individual como no coletivo, tendo o medicamento como insumo essencial (Filho \& Barreira, 2017).

A orientação farmacêutica pode proporcionar tanto benefícios ao portador quanto ao cuidador, medida que os benefícios do acompanhamento farmacoterapêutico atingem a ambos reduzindo custos, melhorando as prescrições, promovendo maior adesão do portador ao tratamento e controlando a possibilidade de reações adversas (Filho \& Barreira, 2017).

O farmacêutico possui a função de informar os cuidadores ou portadores com Doença de Alzheimer, em fase inicial da patologia, e ainda independentes, a realizar o acompanhamento farmacoterapêutico, estando atentos às reações adversas aos medicamentos e as interações medicamentosas, para assegurar a eficácia e segurança da terapêutica em cada um dos estágios, na progressão da patologia, no aparecimento das comorbidades ou enfermidades, mesmo que não relacionadas a esta doença (Fernandes \& Andrade, 2017).

No entanto, através de estudos, observou-se que a orientação farmacêutica, proporciona melhoria da farmacoterapia, tendo em vista que o fármaco é um instrumento primordial de recuperação e manutenção da saúde dos portadores com Alzheimer. A averiguação da farmacoterapia em idosos afetados, assim como de seus cuidadores, é um instrumento fundamental de avaliação da qualidade da atenção fornecida a estes indivíduos. Esforços para aperfeiçoar a prescrição seleção, a dispensação e o emprego de medicamentos devem estabelecer prioridade nos programas de atenção ao idoso (Filho \& Barreira, 2017).

\section{Considerações Finais}

Haja visto a necessidade recorrente do profissional farmacêutico na maioria das patologias, em relação a DA esta necessidade está evidente, pois os dados apresentados apontam que para que se tenha uma real e eficaz adesão ao tratamento 
medicamentoso é de suma importância uma multidisciplinariedade no acompanhamento do tratamento. Nesse contexto, os imunomoduladores têm maior probabilidade de se tornarem drogas capazes de influenciar o curso da DA, pois os estudos selecionados apresentaram melhor qualidade e os resultados foram promissores. No entanto, a toxicidade dessas drogas para o tratamento da DA constitui um grande obstáculo, e para contornar tal situação a presença do profissional farmacêutico se torna cada vez mais imprescindível.

\section{Referências}

Allegri, R. F. (2020). Mudar de demências neurodegenerativas para proteinopatias cognitivas, substituindo "onde" por "o quê". Dement. Neuropsicol, 14(3), 237-242. Disponível em: https://www.scielo.br/scielo.php?script=sci_arttext\&pid=S1980-57642020000300237\&lang=pt.

Almeida, O. (1998). Tratamento da doença de Alzheimer: avaliação crítica sobre o uso de anticolinesterásicos. Arq. Neuro-Psiquiatr., 56(3), 688-696. Disponível em: https://www.scielo.br/scielo.php?script=sci_arttext\&pid=S0004-282X1998000400029\&lang=pt.

Almeida-brasil, C. C., et al. (2016). Acesso aos medicamentos para tratamento da doença de Alzheimer fornecidos pelo Sistema Único de Saúde em Minas Gerais, Brasil. Cad. Saúde Pública, 32(7). Disponível em: https://www.scielo.br/scielo.php?script=sci_arttext\&pid=S0102-311X2016000705003\&lang=pt

Antunes, J. A. P. J. (2017). Refugees and mental health - hosting, understand and treat, Portugal, 18(1). Disponível em: http://www.scielo.mec.pt/scielo.php?script=sci_arttext\&pid=S1645-00862017000100010\&lng=pt\&nrm=iso

Araujo, C. E. P.; Tascarollo, I. L.; \& Antonio, M.A (2019). Farmácia Clínica e Atenção Farmacêutica. Paraná: Atena Editora. Disponível em: https://www.finersistemas.com/atenaeditora/index.php/admin/api/artigoPDF/27499

Brasil. (2017). Protocolo Clínico e Diretrizes Terapêuticas da Doença de Alzheimer.

Carvalho, P. D. P.; magalhaes, C. M. C.; \& Pedroso, J. S. (2016). Tratamentos não farmacológicos que melhoram a qualidade de vida de idosos com doença de Alzheimer: uma revisão sistemática. J. bras. psiquiatr., Rio de Janeiro, 65(4), 334-339. Disponível em: https://www.scielo.br/scielo.php?script=sci_arttext\&pid=S0047-20852016000400334\&lang=pt.

Cazarim, M. S., et al. (2016). Perspectivas para o tratamento da doença de Alzheimer: uma revisão sobre substâncias farmacológicas promissoras. São Paulo Med. J, 134(4), 342-354. Disponível em: https://www.scielo.br/scielo.php?script=sci_arttext\&pid=S1516-31802016000400342\&lang=pt.

Fernandes, J. S. G.; \& Andrade, M. S. (2017). Revisão sobre a doença de alzheimer: diagnóstico, evolução e cuidados. Psicologia, Saúde \& Doenças, 18(1), 131-140. Diponível em: http://www.scielo.mec.pt/scielo.php?script=sci_arttext\&pid=S1645-00862017000100011.

Ferreira, S.; \& Massano, J. (2013). Terapêutica farmacológica na doença de Alzheimer: progressos e esperanças futuras, Porto, 27(2), 2-4. Disponível em: http://www.scielo.mec.pt/scielo.php?script=sci_arttext\&pid=S0871-34132013000200004.

Filho, R. P. B.; \& Barreira, I.V.B.P. (2017). Doença de Alzheimer: diagnóstico e perspectiva. Rio de Janeiro: Gramma Livraria e Editora. Disponível em: https://books.google.com.br/books?id=F9BBDwAAQBAJ\&printsec=frontcover\&dq=Doen\%C3\%A7a+de+ALZHEIMER\&hl=pt-

BR\&sa=X\&ved=2ahUKEwjE-fKEiq7tAhWLLLkGHSUYC4sQ6AEwAHoECAQQAg\#v=onepage\&q=Doen\%C3\%A7a\%20de\%20ALZHEIMER\&f=false.

Madureira, B. G., et al. (2018). Efeitos de programas de reabilitação multidisciplinar no tratamento de pacientes com doença de Alzheimer: uma revisão sistemática. Cad. saúde colet., Rio de Janeiro, 26(2), 222-232. Disponível em: https://www.scielo.br/scielo.php?script=sci_arttext\&pid=S1414462X2018000200222\&lang=pt\#B006

Martins, G., et al. (2019). Características sociodemográficas e de saúde de cuidadores formais e informais de idosos com Doença de Alzheimer. Esc. Anna Nery, 23(2), 4-8. Disponível em: https://www.scielo.br/scielo.php?pid=S1414-81452019000200220\&script=sci_arttext\&tlng=pt.

Moreira, S. C.; Jansen, A. K.; \& Silva, F. M. (2020). Intervenções dietéticas e cognição de pacientes com doença de Alzheimer: uma revisão sistemática de ensaio clínico randomizado. Dement. Neuropsicol,, 14(3), 258-282. Disponível em: https://www.scielo.br/scielo.php?script=sci_arttext\&pid=S1980$57642020000300258 \&$ lang=pt.

Pereira, G. C. M.; \& Oliveira, G. C. (2019). Prevalência da síndrome de Capgras em pacientes com Alzheimer: uma revisão sistemática e metaanálise. Dement. neuropsicol, 13(4), 463-468. Disponível em: https://www.scielo.br/scielo.php?script=sci_arttext\&pid=S1980-57642019000400463\&lang=pt. 